\title{
Prevalencia de agentes etiológicos de meningitis bacteriana en población adulta en el Hospital San Martín de Quillota entre los años 2012-2018 Prevalence of etiological agents of acute bacterial meningitis in adult population at the Quillota's San Martin Hospital between years 2012-2018.
}

Juan Pablo Scarella L. ${ }^{1}$, José Andrés Cubillos M. ${ }^{1}$, Camila Garrido P. ${ }^{1}$, Valentina Briceño S. ${ }^{1}$, Dr. José Armijo M. ${ }^{2}$, Dr. Felipe Martínez L. ${ }^{3}$

Introduction: Acute bacterial meningitis (ABM) is denifed as the inflammation of leptomeninges due to the presence of pyogenic bacteriae. Epidemiology is variable and multifactorial. In spite of advance in medicine, ABM's mortality has remained stable, hence the need to study the causative microorganisms in order to guide prevention and treatment strategies. Objectives: To determine the prevalence of the etiological agents of ABM confirmed in adults in a medium complexity hospital located in Quillota, Chile, between 2012 and 2018, to establish the rate of resistance of these to antibiotics. Determine the profile of the patients and establish the relationship between profile, etiological agent identified and outcome. Methods: A sample of 38 cases with ABM was evaluated during, the prevalence of the pathogens involved was established and a statistical analysis was carried out considering the risk factors of patients, their days of in-hospital stay and their condition at hospital discharge. Results: We analyzed 38 cases, 15 without isolated pathogens, 13 of Streptococcus pneumoniae, 4 of Haemophilus spp., 3 of Neisseria meningitidis, 2 of Staphylococcus spp. and we obtained one case of Mycobacterium tuberculosis. In the group without isolated pathogens, a better prognosis was observed, lower blood CRP values and higher glucose on spinal fluid values. 6 deaths per ABM were recorded. Conclusion: The prevalence of etiological agents was similar to that described in the literature, with pneumococcus as the most frequent microorganism, no cases of Listeria monocytogenes were reported. Probably in the group in which no agent was isolated, cases of viral meningitis were included.

Key words: Meningitis, etiology, bacterial agents, prognosis, survival, epidemiology Rev Chil Neuro-Psiquiat 2019; 57 (4): 347-356

Aceptado: 07/01/2020

Recibido: 26/07/2019

1 Interno(a) de Medicina. Universidad Andrés Bello, sede Viña del Mar.

2 Médico Neurólogo, Docente Facultad de Medicina Universidad Andrés Bello, sede Viña del Mar.

Médico Internista, Docente Facultad de Medicina Universidad Andrés Bello, sede Viña del Mar. 


\section{Introducción}

La Meningitis Bacteriana Aguda (MBA) corresponde a la inflamación de las leptomeninges por presencia de bacterias piógenas. Constituye una emergencia médica donde el diagnóstico y tratamiento precoz puede reducir considerablemente su morbimortalidad $^{(1)(2) .}$

La epidemiología es variable y depende de múltiples factores tales como la edad, el agente involucrado y de la presencia de ciertos factores de riesgo ${ }^{(3)}$. En los últimos 30 años, la epidemiología de la MBA ha cambiado sustancialmente, tanto en los patógenos causantes como en los grupos etarios afectados. Estos cambios se deben en gran parte a la implementación de vacunas conjugadas contra Haemophilus influenzae tipo $\mathrm{b}$, Streptococcus pneumoniae (neumococo) y Neisseria meningitidis (meningococo). En Estados Unidos de América (UEA) y Europa la tasa de incidencia varía entre $0,1-0,7$ por cada 100.000 personas. Actualmente, en adultos la primera causa es el neumococo, seguido por el meningococo, siendo la tercera en frecuencia Listeria monocytogenes (4), comúnmente encontrada en pacientes con ciertos factores de riesgo como edad mayor a 50 años, inmunodeficiencias, alcoholismo, cáncer y terapia inmunosupresora (5). La mayoría de los casos de MBA son de adquisición comunitaria, mientras que otras bacterias como Staphylococcus spp. y bacilos Gram negativos se presentan en contexto nosocomial o secundario a trauma ${ }^{(6)}$.

La resistencia antibiótica suele ser baja. En el estudio de Diederik Van Beek et. al del año 2004 se constató una resistencia inmediata a penicilina de un $0,36 \%$ en cepas neumocócicas y 1,56\% en meningocócicas. Estos resultados son concordantes con otro estudio del mismo autor del $2002^{(7)(8)}$. Sin embargo, en UEA, Francia, España y otros países, la tasa de resistencia reportada en neumococo es mayor ( $24 \%$ en promedio) ${ }^{(9)}$.
Las manifestaciones clínicas de la MBA van a depender de múltiples factores, tales como la edad del paciente y el microorganismo involucrado ${ }^{(2)}$. La tríada clásica comprende fiebre, cefalea holocraneana asociada a rigidez de cuello y compromiso de conciencia. Un estudio realizado en los Países Bajos, evidenció dicha tríada en solo el $41 \%$ de los casos. Otros síntomas asociados pueden ser déficits neurológicos focales, convulsiones o parálisis de nervios craneales. Además, puede haber manifestaciones cutáneas como la erupción petequial, un signo característico de la bacteremia por meningococo, pero que también puede estar presente en meningitis neumocócicas ${ }^{(1)}$.

En el estudio diagnóstico de la MBA se incluye habitualmente hemocultivos, neuroimágenes y la punción lumbar con análisis del LCR que se lleva el rol protagónico, e incluye el estudio citoquímico, tinción de Gram, cultivo y en ocasiones otras pruebas más específicas como los métodos de biología molecular. Es importante destacar que cuando existe una sospecha bien fundada el estudio diagnóstico no debe retrasar el inicio de la terapia antibiótica empírica (habitualmente con Cefalosporinas de tercera generación), ya que se ha demostrado que este retraso genera un aumento en la morbimortalidad $^{(1)(10)(11) .}$

Según una revisión Cochrane del 2015 estaría recomendado el uso corticoides como terapia coadyudante, dado que se vio una reducción significativa en las potenciales secuelas neurológicas ${ }^{(1)(12)(13)(14) \text {. }}$

A pesar de los progresos en la medicina intensiva y amplia gama de antibióticos disponibles, un estudio realizado en EUA donde se analizaron datos de meningitis bacteriana en adultos entre los años 1998 y 2007 , evidenció que la mortalidad de la MBA se ha mantenido relativamente estable durante las últimas décadas ${ }^{(15)}$, de ahí radica la necesidad de estudiar los microorganismos causantes para poder orientar estrategias de 
prevención y tratamiento ${ }^{(4)}$, esto cobra mayor importancia en nuestro país dado que no se encontraron estudios locales sobre esta materia. Es por esto que en este trabajo el objetivo principal consiste en determinar la prevalencia de los agentes etiológicos de MBA confirmadas en adultos en el HSMQ entre los años 2012 y 2018; y a su vez establecer la tasa de resistencia de éstos a antibióticos de uso habitual. También se busca determinar el perfil de los pacientes incluidos en la muestra según sexo, edad y factores de riesgo, y establecer la relación entre este perfil, el agente etiológico identificado y el desenlace de cada paciente.

\section{Metodología}

Previo a la realización de la investigación se solicitó la aprobación del Comité de Bioética de la Facultad de Medicina de la Universidad Andrés Bello Viña del Mar y la autorización de la dirección del HSMQ.

Se realizó un estudio observacional de cohorte retrospectiva que se estructuró en base a las pautas STROBE (Strengthening the Reporting of Observational studies in Epidemiology) ${ }^{(16)}$. Se solicitó al Grupo Relacionado al Diagnóstico (GRD) del Departamento de Control de Gestión del HSMQ el número de ficha, los días de estadía, la condición al egreso (vivo, muerto) y destino al egreso (domicilio o traslado) de los pacientes con edad mayor o igual a 16 años hospitalizados entre los años 2012 y 2018. Luego se pidieron las fichas de dichos pacientes a través de la Subdirección Médica del HSMQ y se realizó una revisión de éstas.

Los criterios de inclusión fueron: pacientes con edad igual o mayor de 16 años que hayan permanecido hospitalizados en HSMQ entre los años 2012 y 2018, y que cuenten con una MBA diagnosticada mediante tinción de Gram o cultivo de LCR. En caso de que no se haya aislado patógeno en los estudios mencionados se consideró como MBA los casos que cumplieran con dos o más de los siguientes criterios: glóbulos blancos en LCR mayor a 100 por $\mathrm{mm}$, glucorraquia menor a $40 \mathrm{mg} / \mathrm{dl}$ o menor a $40 \%$ de glicemia, proteinorraquia mayor a $45 \mathrm{mg} / \mathrm{dL}$ y diagnóstico clínico de MBA en epicrisis de hospitalización ${ }^{(18)}$.

Se excluyeron del estudio los casos con diagnóstico de MBA asociada a la atención en salud, meningitis virales, antecedentes de neurocirugía reciente (hace menos de 6 meses) y pacientes sin un estudio de LCR confirmatorio.

Una vez seleccionados los pacientes, se recopilaron datos de la hospitalización tales como: edad, sexo, comorbilidades, embarazo al momento del diagnóstico, estudio del LCR, patógeno detectado mediante tinción de Gram o cultivo, antibiograma asociado, tratamiento recibido, proteína $\mathrm{C}$ reactiva $\mathrm{y}$ recuento de leucocitos en sangre periférica al ingreso. Con esta información, sumada a la aportada por el GRD, se elaboró una base de datos y se realizó un análisis retrospectivo, considerando el tiempo de seguimiento como el transcurrido desde el ingreso del paciente a la Unidad de Emergencia de Adultos del HSMQ hasta el egreso hospitalario.

Para el análisis descriptivo de variables cuantitativas se utilizaron: media, mediana, desviación estándar y rango intercuartil; para variables cualitativas: frecuencias absolutas y relativas. Por otro lado, en el análisis inferencial se utilizó la Prueba Exacto de Fisher en busca de asociaciones entre variables cualitativas, Prueba de Mann-Whitney para la comparación de medias y Curvas de Supervivencia de Kaplan Meier para evaluar variables de desenlace. Se utilizó el software SPSS v15 para Windows 7. 


\section{Resultados}

Caracterización general: se solicitaron 55 fichas clínicas de las cuales se recibieron 51, ya que 4 de estas no se encontraban disponibles en el archivo del HSMQ. Se revisaron las fichas, aplicándose criterios de inclusión y exclusión, descartando en total 17: 9 no contaban con diagnóstico de MBA (error de registro o hipótesis diagnóstica inicial que se descartó), 2 fueron tratamiento empírico con punción lumbar no realizada o frustra y 2 por neurocirugía reciente. Debido a esto, se estudiaron 38 casos, a partir de los cuales se generó una base de datos y se realizó el análisis estadístico.

Caracterización del perfil de pacientes: la mitad de los casos ${ }^{(19)}$ correspondieron a pacientes de género femenino. La edad media fue de 47 años (DS 18,17). En cuanto a los factores de riesgo estudiados se observó 1 caso de infección por Virus de la Inmunodeficiencia Humana (VIH), 3 de alcoholismo, 10 de Diabetes Mellitus 2 y 10 mayores de 60 años. No se encontraron pacientes embarazadas (tabla 1).

Tabla 1: Caracterización de pacientes con MBA confirmadas en adultos en el HSMQ entre los años 2012 y 2018

\begin{tabular}{|c|c|c|c|c|}
\hline & $\mathbf{N}$ & Media & Desviación estándar & Intervalo de confianza $95 \%$ \\
\hline Edad & 38 & 46,74 & 18,17 & $40,76-52,71$ \\
\hline RGB en LCR & 14 & 4420,43 & 7431,67 & $129,50-8711,35$ \\
\hline Proteinorraquia & 24 & 314,27 & 287,05 & $193,06-435,49$ \\
\hline Glucorraquia & 35 & 30,55 & 31,97 & $19,57-41,53$ \\
\hline PCR en sangre & 38 & 145,71 & 122,30 & $-185,915$ \\
\hline RGB en sangre & 38 & 18410,3 & 9800,43 & $15189,2-21631,85$ \\
\hline Días de estada & 38 & 15,53 & 8,58 & $12,70 \quad-18,35$ \\
\hline
\end{tabular}

Prevalencia de agentes etiológicos: en $\mathbf{1 5}$ casos no se aisló patógeno en LCR, en el resto de los casos se encontraron 13 MBA porneumococo, 4 por Haemophilus spp, 3 por meningococo, 2 por Staphylococcus spp y 1 por Mycobacterium tuberculosis. No se obtuvo ningún caso de Listeria monocytogenes (tabla 2).

Prevalencia de antibióticos utilizados: respecto a los antibióticos utilizados, se observó que las Cefalosporinas de $3^{\mathrm{a}}$ generación fueron utilizadas en 34 casos, en 12 casos de asoció Ampicilina y en 2 Vancomicina. En 4 pacientes se utilizaron otros antibióticos para el manejo de MBA. Por otra parte, se encontraron 3 casos de resistencia a Cefa- losporinas de tercera generación, 2 fueron Staphylococcus spp. y 1 caso de neumococo (tabla 2).

Caracterización del Pronóstico: se aplicaron las curvas de supervivencia de KaplanMeier según edad mayor o menor a 60 años respecto a los días de estadía, donde se obtuvo una relación estadísticamente significativa $(p<0,00)$, que mostró que los pacientes mayores de 60 años presentaron una estadía. En cambio los pacientes menores de 60 años presentaban hospitalizaciones más prolongadas, con fallecimientos sobre los 28 días de hospitalización (figura 1). Por otro lado se realizaron curvas de supervivencia de Kaplan-Meier según agentes aislados y los 
días de estadía. Se aplicó la prueba de Log Rank donde se obtuvo que los días de estadía según los agentes aislados y no aislados, no fueron estadísticamente significativos $(\mathrm{p}=$ 0,278) (figura 2).

Tabla 2: Prevalencia de los agentes etiológicos de MBA confirmadas en adultos en el HSMQ entre los años 2012 y 2018

\begin{tabular}{|l|c|c|c|}
\hline Agentes etiologicos MBA & Frecuencia & Porcentaje & Intervalo de Confianza 95\% \\
\hline Patógeno no aislado & 15 & 39,5 & $25,60-55,30$ \\
\hline Streptococcus pneumoniae & 13 & 34,2 & $21,21-50,10$ \\
\hline Haemophilus spp & 4 & 10,5 & $4,17-50,10$ \\
\hline Neisseria meningitidis & 3 & 7,9 & $2,72-20,80$ \\
\hline $\begin{array}{l}\text { Staphylococcus spp } \\
\text { Mycobacterium }\end{array}$ & 2 & 5,3 & $1,46-17,29$ \\
tuberculosis & 1 & 2,6 & $0,47-13,49$ \\
Total & 38 & 100 & \\
\hline
\end{tabular}

Figura 1: Curvas de supervivencia de Kaplan Meier según edad mayor o menor a 60 años

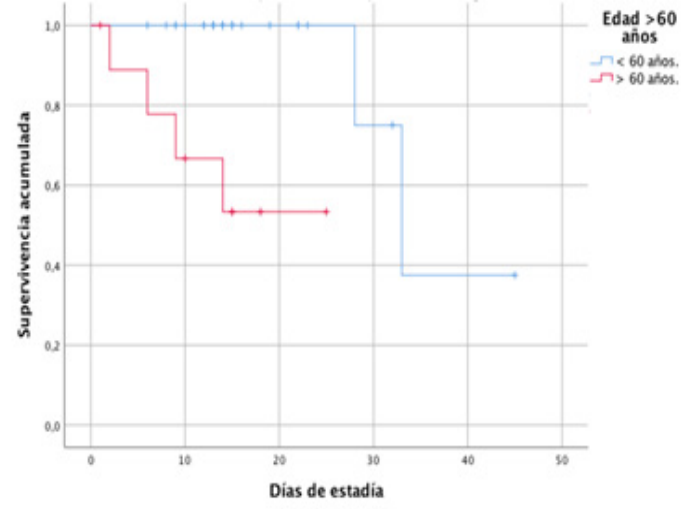

También se analizaron los datos respecto a los valores de Glucorraquia en LCR y la presencia de Agente aislado y no aislado. Se aplicó la prueba de Mann-Whitney, donde se obtuvo una relación estadísticamente significativa $(\mathrm{p}=0,001)$, que mostraba una glucorraquia más baja con un rango de dispersión menor en el grupo de agentes aislados versus no aislado (figura 3 ).

En cuanto a los valores de PCR en sangre entre los grupos de casos con agentes bacterianos aislados y no aislados en LCR, se aplicó la prueba de Mann-Whitney, obteniéndose una relación estadísticamente sig-
Figura 2: Curvas de supervivencia de Kaplan Meier según agentes aislados

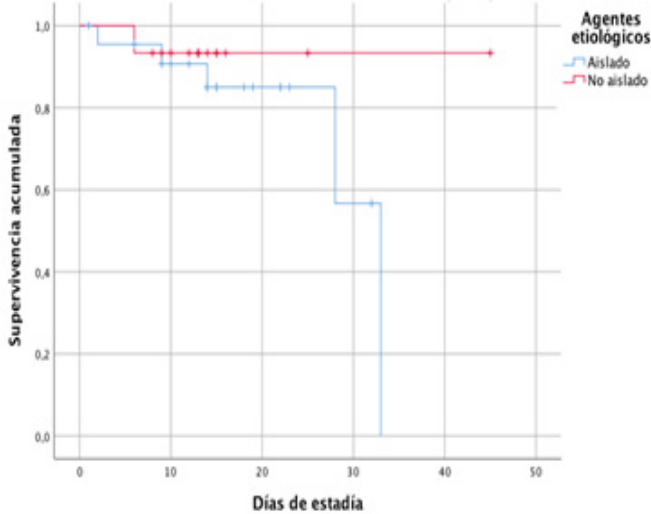

nificativa $(\mathrm{p}=0,008)$ donde los valores más bajos se presentaban en pacientes con agentes no aislados (figura 4).

Caracterización de condición de egreso hospitalario: de los 38 casos, 32 fueron dados de alta vivos a su domicilio y 6 murieron en contexto de su MBA, de estos 2 casos fueron por neumococo, 2 por Staphylococcus aureus, 1 por Mycobacterium tuberculosis y por último 1 caso sin microorganismo aislado. 
Figura 3: Diagrama de cajas y bigotes de glucorraquia por agente aislado y no aislado

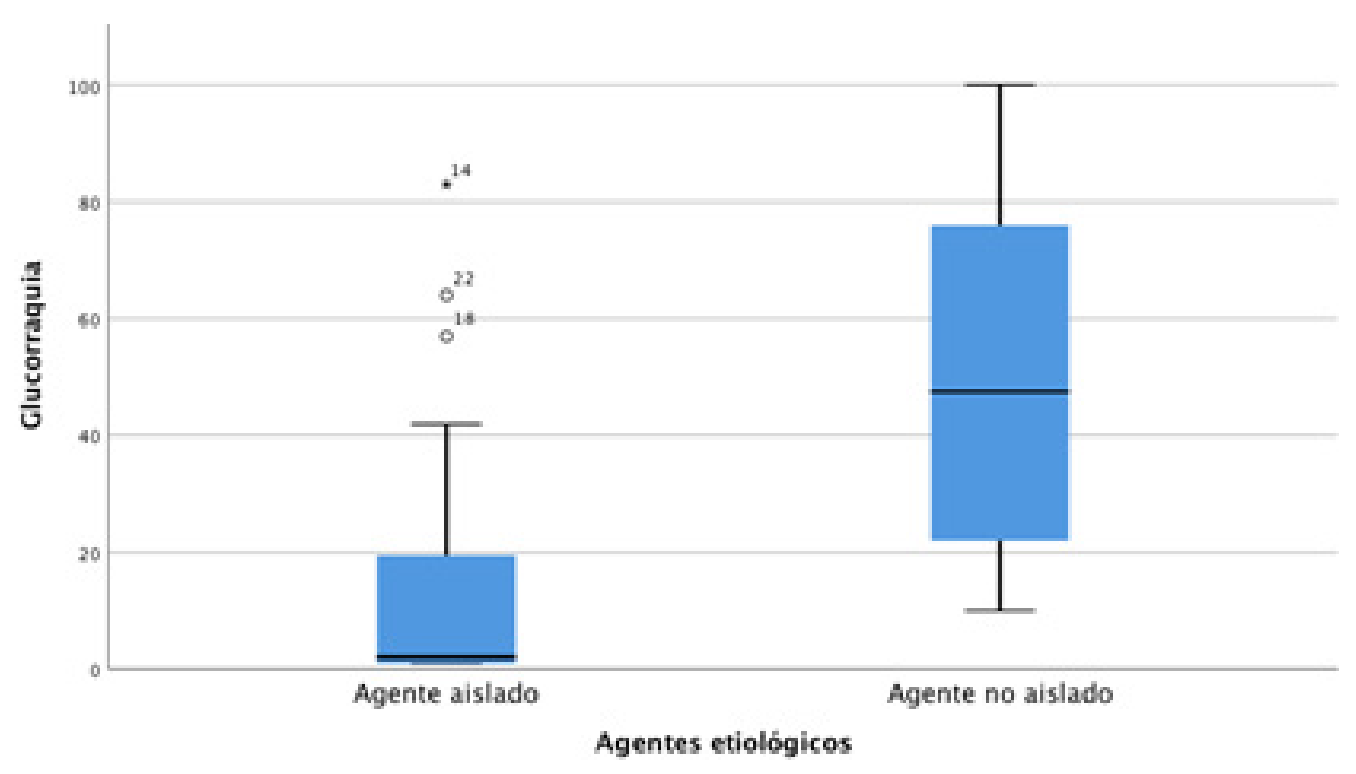

Figura 4: Diagrama de cajas y bigotes de PCR en sangre por agentes aislados y no aislados en LCR

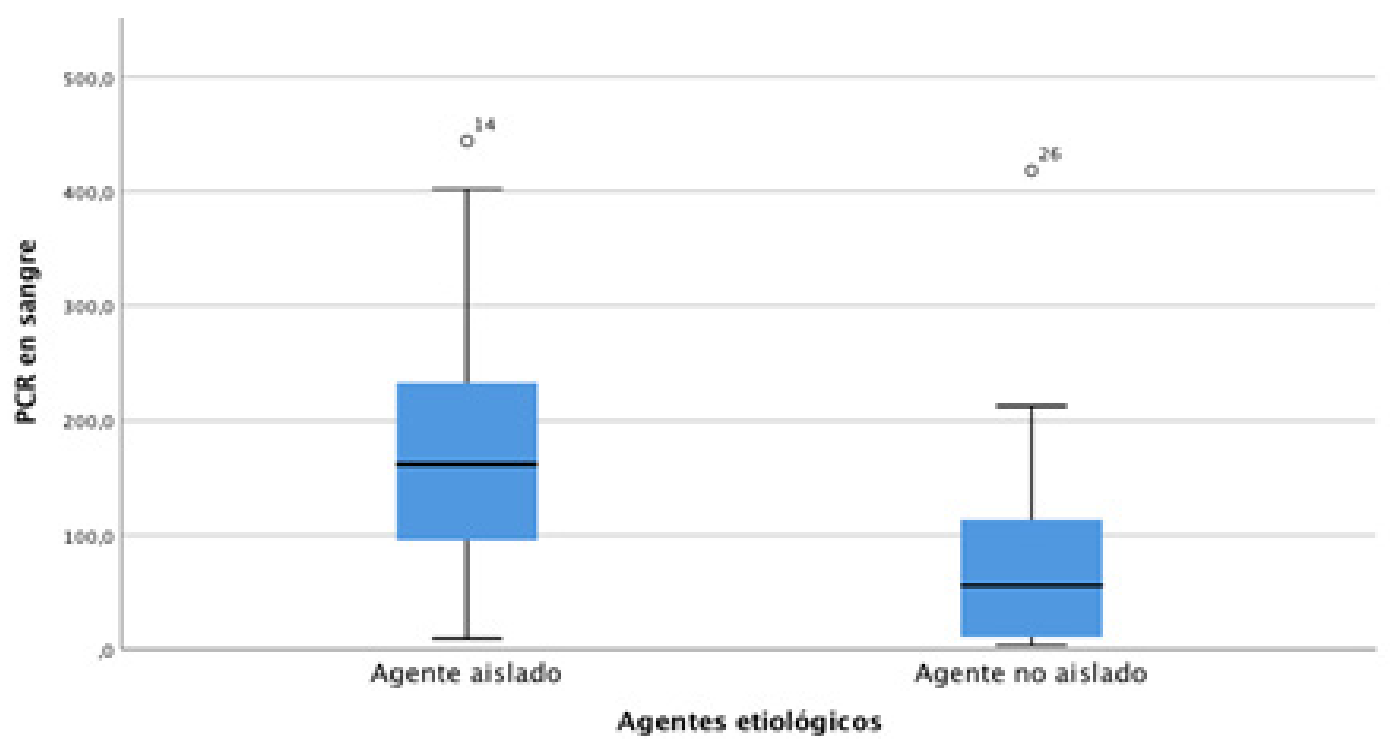

\section{Discusión}

Se logró incluir un total de 38 casos de MBA confirmadas en adultos en el HSMQ entre los años 2012 y 2018 . Dado el reducido tamaño muestral obtenido, se utilizaron pruebas no paramétricas, pero la información obtenida es relevante para comprender de mejor manera la realidad local.
En cuanto a la prevalencia de agentes bacterianos, el neumococo fue el microorganismo más frecuente (13 casos) lo que coincide con las series internacionales revisadas. Por otro lado, se encontraron 4 casos de Haemophilus spp., 3 de meningococo y 2 de Staphylococcus spp., lo que también se condice con el conocimiento actual. No se registraron casos de Listeria monocytoge- 
nes, a pesar de que se incluyeron pacientes con factores de riesgo para desarrollar este tipo de MBA y de que ocupa alrededor del 3 puesto en frecuencia en las series revisadas. Esta ausencia puede atribuirse al tamaño muestral reducido, a la falta de cultivos específicos (no incluidos en la orden de exámenes del HSMQ previo al año 2015) y que la tinción de Gram y cultivo del LCR tienen una sensibilidad entre $70-85 \%{ }^{(17)}$.

El mayor grupo lo constituyeron casos sin agentes bacterianos aislados. Si bien podrían existir distintas causas que expliquen este fenómeno, como el uso de tratamientos previos y la falta de sensibilidad de los exámenes de laboratorio y cultivos, al realizar los diferentes análisis, comparando las demás variables se obtuvo que los valores de glucorraquia en el grupo en que no se aisló patógeno resultaron significativamente mayores $(p=0,001)$, los valores de PCR en sangre fueron significativamente menores $(p=0,008)$ y además mostraron una menor mortalidad $(\mathrm{p}=0,00)$, por lo tanto, se podría proponer que una parte no menor de dichos casos corresponderían a meningitis virales. Considerando que el tratamiento de la meningitis viral es sintomático, el sobretratamiento con antibióticos y corticoides no debiera significar un gran problema, además que ante la sospecha fundada de MBA se recomienda tratar como tal. Sin embargo, hace cuestionarse los criterios de inclusión, la falta de precisión en el proceso diagnóstico y la necesidad de incorporar nuevas estrategias de laboratorio que excluyan con mayor certeza el diagnóstico de MBA o identifiquen de la manera más sensible y específica los agentes virales.

Los antibióticos utilizados fueron principalmente las Cefalosporinas de tercera generación (34 casos), en 12 casos se asoció a Ampicilina y sólo 2 a Vancomicina, lo que se condice con las recomendaciones nacionales e internacionales del tratamiento de la MBA.

Para estudiar los rangos etarios se separó la muestra en dos grupos: mayores y menores de 60 años. Se observó que los pacientes del grupo de mayor edad presentaban una mortalidad mayor $(p=0,00)$ y más precoz (antes de los 20 días de hospitalización), lo que por un lado sería esperable y por otro, permitiría explicar que las estadías hospitalarias de los más jóvenes sean mayores.

El pronóstico general fue favorable. Solo 6 de los 38 casos $(15,8 \%)$ fallecieron por MBA, esto podría explicarse en parte por la posibilidad de que dentro del grupo en que no se aisló patógeno, existieran meningitis virales. En dicho grupo solo se registró un fallecimiento. Por otro lado, dado el reducido tamaño muestral, no se pudieron plantear estadísticas representativas de mortalidad por MBA.

Una de las limitaciones de este trabajo es que no se consideraron las eventuales secuelas de la MBA, lo que podría plantearse para una nueva investigación con una muestra mayor.

\section{Conclusión}

La prevalencia de los agentes etiológicos de MBA fue similar a la descrita en la literatura, destacándose $\mathrm{S}$. pneumoniae como el microorganismo más frecuente y ausencia de L. monocytogenes. Por otro lado, en un gran número de los casos no se aisló microorganismo y luego de realizar el análisis se infirió que algunos de estos podrían corresponder a meningitis virales.

Esta investigación podría ser utilizada como punto de comparación y base de futuros estudios locales, en los que se podrían incluir un mayor número de casos, para representar de mejor forma la realidad de la MBA nivel regional y nacional, para poder establecer nuevos protocolos y estrategias de manejo, en busca de disminuir su morbimortalidad. 


\begin{abstract}
Resumen
Introducción: La Meningitis Bacteriana Aguda (MBA) corresponde a la inflamación de las leptomeninges por presencia de bacterias piógenas. La epidemiología es variable y depende multifactorial. A pesar de los avances en medicina la mortalidad de la MBA se ha mantenido estable, evidenciando la necesidad de estudiar esta patología para orientar estrategias de prevención y tratamiento. Objetivos: Determinar la prevalencia de los agentes etiológicos de MBA confirmadas en adultos en el Hospital San Martín de Quillota (HSMQ) entre los años 2012 y 2018. Establecer la tasa de resistencia a antibióticos. Determinar el perfil de los pacientes, y establecer la relación entre perfil, agente etiológico identificado y desenlace. Métodos: c muestra de 38 casos, se estableció la prevalencia de los patógenos involucrados y se realizó un análisis estadístico considerando los factores de riesgo, sus días de estadía y su condición al egreso hospitalario. Resultados: 15 casos en que no se aisló patógeno en LCR (líquido cefalorraquideo), 13 MBA por neumococo, 4 por Haemophilus spp, 3 por meningococo, 2 por Staphylococcus spp y 1 por Mycobacterium tuberculosis. En el grupo sin agente aislado se observó un mejor pronóstico, valores de PCR (proteína $C$ reactiva) en sangre menores y valores de glucorraquia mayores. Se registraron 6 fallecimientos por MBA. Conclusión: La prevalencia de los agentes etiológicos fue similar a la descrita en la literatura, con neumococo como el microorganismo más frecuente, no se reportaron casos de Listeria monocytogenes. Probablemente en el grupo sin agente aislado se incluyeron casos de meningitis virales.

Palabras clave: Meningitis, etiología, agentes bacterianos, pronóstico, supervivencia, epidemiología.
\end{abstract}

\section{Referencias Bibliográficas}

1. van de Beek D, Brouwer M, Hasbun R, Koedel U, Whitney CG, Wijdicks E. Community-acquired bacterial meningitis. Nat Rev Dis Prim [Internet]. 2016;2:16074. Available from: http://www.ncbi.nlm.nih.gov/pubmed/27808261.

2. Figueiredo AHA, Brouwer MC, van de Beek D. Acute Community-Acquired Bacterial Meningitis. Neurol Clin [Internet]. 2018 Nov;36(4):809-20. Available from: http://www.ncbi.nlm. nih.gov/pubmed/30366556.

3. Swartz MN. Bacterial meningitis--a view of the past 90 years. N Engl J Med [Internet]. 2004 Oct 28;351(18):18268. Available from: http://www.ncbi. nlm.nih.gov/pubmed/15509815
4. Conca N, Santolaya ME, Farfan MJ, Cofré F, Vergara A, Salazar L, et al. Diagnóstico etiológico en meningitis y encefalitis por técnicas de biología molecular. Rev Chil Pediatría [Internet]. 2016 Jan;87(1):24-30. Available from: http://www.scielo. cl/scielo.php?script=sci_arttext\&pi$\mathrm{d}=$ S0370-41062016000100005\&lan$\mathrm{g}=\mathrm{pt} \% 5 \mathrm{Cnhttp}: / /$ www.scielo.cl/pdf/ $\mathrm{rcp} / \mathrm{v} 87 \mathrm{n1} / \mathrm{art} 05 . \mathrm{pdf}$

5. Lundbo LF, Benfield T. Risk factors for community-acquired bacterial meningitis. Infect Dis (London, England) [Internet]. 2017 Jun;49(6):433-44. Available from: http://www.ncbi.nlm. nih.gov/pubmed/28301990.

6. Rodrigo Blamey D. Meningitis bacteriana aguda. Rev Médica Clínica Las Condes [Internet]. 2014 
May;25(3):534-40. Available from: https://linkinghub.elsevier.com/retrieve/pii/S0716864014700677.

7. van de Beek D, de Gans J, Spanjaard L, Weisfelt M, Reitsma JB, Vermeulen M. Clinical features and prognostic factors in adults with bacterial meningitis. N Engl J Med [Internet]. 2004 Oct 28;351(18):1849-59. Available from: http://www.ncbi.nlm.nih.gov/ pubmed/15509818.

8. van de Beek D, de Gans J, Spanjaard L, Vermeulen M, Dankert J. Antibiotic guidelines and antibiotic use in adult bacterial meningitis in The Netherlands. J Antimicrob Chemother [Internet]. 2002 Apr;49(4):661-6. Available from: http://www.ncbi.nlm.nih.gov/ pubmed/11909840.

9. Whitney CG, Farley MM, Hadler J, Harrison LH, Lexau C, Reingold A, et al. Increasing prevalence of multidrug-resistant Streptococcus pneumoniae in the United States. N Engl J Med [Internet]. $2000 \operatorname{Dec} 28 ; 343(26): 1917$ 24. Available from: http://www.ncbi. nlm.nih.gov/pubmed/11136262.

10. Auburtin M, Wolff M, Charpentier J, Varon E, Le Tulzo Y, Girault C, et al. Detrimental role of delayed antibiotic administration and penicillin-nonsusceptible strains in adult intensive care unit patients with pneumococcal meningitis: the PNEUMOREA prospective multicenter study. Crit Care Med [Internet]. 2006 Nov;34(11):2758-65. Available from: http://www.ncbi.nlm. nih.gov/pubmed/16915106.

11. Lepur D, Barsić B. Community-acquired bacterial meningitis in adults: antibiotic timing in disease course and outcome. Infection [Internet]. 2007 Jun;35(4):225-31. Available from: http://www.ncbi.nlm.nih.gov/pub$\mathrm{med} / 17646915$

12. Brouwer MC, McIntyre P, Prasad
K, van de Beek D. Corticosteroids for acute bacterial meningitis. Cochrane database Syst Rev [Internet]. 2015 Sep 12;3(9):CD004405. Available from: http://www.ncbi.nlm.nih.gov/pub$\mathrm{med} / 26362566$.

13. van Ettekoven $\mathrm{CN}$, van de Beek $\mathrm{D}$, Brouwer MC. Update on community-acquired bacterial meningitis: guidance and challenges. Clin Microbiol Infect [Internet]. 2017 Sep;23(9):6016. Available from: http://www.ncbi. nlm.nih.gov/pubmed/28478238.

14. Glimåker $\mathrm{M}$, Johansson $\mathrm{B}$, Grindborg Ö, Bottai M, Lindquist L, Sjölin J. Adult bacterial meningitis: earlier treatment and improved outcome following guideline revision promoting prompt lumbar puncture. Clin Infect Dis [Internet]. 2015 Apr 15;60(8):1162-9. Available from: http://www.ncbi.nlm. nih.gov/pubmed/25663160.

15. Thigpen MC, Whitney CG, Messonnier NE, Zell ER, Lynfield R, Hadler JL, et al. Bacterial meningitis in the United States, 1998-2007. N Engl J Med [Internet]. 2011 May 26;364(21):2016-25. Available from: http://www.ncbi.nlm. nih.gov/pubmed/21612470.

16. von Elm E, Altman DG, Egger M, Pocock SJ, Gøtzsche PC, Vandenbroucke JP, et al. The Strengthening the Reporting of Observational Studies in Epidemiology (STROBE) statement: guidelines for reporting observational studies. Lancet (London, England) [Internet]. 2007 Oct 20;370(9596):1453-7. Available from: http://www.ncbi.nlm.nih. gov/pubmed/18064739.

17. Tunkel AR, Hartman BJ, Kaplan SL, Kaufman BA, Roos KL, Scheld WM, et al. Practice guidelines for the management of bacterial meningitis. Clin Infect Dis [Internet]. 2004 Nov 1;39(9):1267-84. Available from: http://www.ncbi.nlm.nih.gov/pub- 
med/15494903.

18. Stephen L. Hauser. Harrison. Neurología en Medicina Clínica. Primera edición en español traducción por Escamilla C. Madrid. McGraw-Hill-Interamericana de España. 1th. Ed. 2006. Capítulo III.

Correspondencia:

Juan Pablo Scarella Larraín

Medicina

Universidad Andrés Bello Viña del Mar

http://facultades.unab.cl/medicina

jpscarella91@gmail.com

(59-9) 84128451 